\title{
Lower expression of endogenous intestinal alkaline phosphatase may predict worse prognosis in patients with Crohn's disease
}

\author{
Seon-Young Park' ${ }^{1 \dagger}$, Ji-Young Kim ${ }^{1 \dagger}$, Su-Mi Lee ${ }^{1}$, Jin Ook Chung ${ }^{2}$, Ji-Ho Seo ${ }^{1}$, SunMin Kim', Dong Hyun Kim', \\ Chang-Hwan Park', Jae-Kyun Ju ${ }^{3}$, Young-Eun Joo' ${ }^{1}$, Jae-Hyuk Lee ${ }^{4}$, Hyun-Soo Kim ${ }^{1,5^{*}}$, Sung-Kyu Choi ${ }^{1}$ and \\ Jong-Sun Rew ${ }^{1}$
}

\begin{abstract}
Backgrounds: Intestinal alkaline phosphatase (IAP) plays important role in gut homeostasis. We aimed to evaluate the expression of endogenous IAP and to assess the clinical course according to the expression of endogenous IAP in patients with Crohn's disease (CD).

Methods: A total of 32 consecutive patients (14 males) with CD were included in the study. We measured the level of endogenous iAP in inflamed and noninflamed colonic mucosa. To verify the inflammation status, we measured the level of mRNA for IL-6, TNF-a, and TLR-4. We monitored the clinical courses of patients during follow-up after acquisition of biopsy specimens.

Results: Median age of patients was 22.5 years (range, 15-49). Median CD activity index (CDAl, range) was 93.7 (22.8 154.9). There were colonic involvements in all patients and perianal involvement in $43.8 \%$ patients. The mRNA levels of IL-6 $(p=0.005)$ and TLR-4 $(p=0.013)$ in inflamed mucosa were significantly higher than those in non-inflamed mucosa. However, there was no difference of expression of TNF-a mRNA $(p=0.345)$. During a 14-month follow-up (range, 9 months-54 months), there were 19 patients with clinical recurrences. There were 9 patients $(9 / 19,47.4 \%)$ with IAP expression ratio (inflamed to non-inflamed) $\leq 1.0$ in patients with clinical recurrence while there was one patient $(1 / 13,7.7 \%)$ with IAP ratio $\leq 1.0$ in patients without clinical recurrence $(p=0.024)$.
\end{abstract}

Conclusion: Lower expression of IAP in inflamed mucosa compared to non-inflamed mucosa may be associated with clinical recurrence in patients with $C D$.

Keywords: Inflammation, Recurrence, Crohn's disease

\section{Background}

Intestinal alkaline phosphatase (IAP) is expressed by brush border of intestinal epithelium [1]. It plays an important role in the interaction between the gut flora and the host by detoxification of bacterial endotoxin, regulation of intestinal microbiome, and regulation of intestinal lipid absorption [2]. Low expression of IAP could increase the

\footnotetext{
* Correspondence: hskim@chonnam.ac.kr; dshskim@jnu.ac.kr

†Seon-Young Park and Ji-Young Kim contributed equally to this work.

${ }^{1}$ Division of Gastroenterology and Hepatology, Chonnam National University

Medical School, Gwangju, South Korea

${ }^{5}$ Department of Internal Medicine, Chonnam National University Medical

School, 42, Jaebongro, Dong-ku, Gwangju 501-757, Korea

Full list of author information is available at the end of the article
}

risk of disease through changes in the microbiome, inflammation, and permeability in intestine. Previous studies showed that low expression of IAP was associated with chronic inflammation-related diseases such as inflammatory bowel disease (IBD), celiac disease, obesity and insulin resistance [3-5]. Moreover, in mouse model with chronic colitis, IAP knockout mouse were more vulnerable to variable stimuli, suggesting protective roles of endogenous IAP for chronic inflammation [6]. Until now, there is limited information for the role of IAP as a predictor for prognosis of patients with Crohn's disease (CD). We hypothesized that low expression of IAP may be associated with poor prognosis in subjects with $C D$. Here, we

(c) The Author(s). 2018 Open Access This article is distributed under the terms of the Creative Commons Attribution 4.0 International License (http://creativecommons.org/licenses/by/4.0/), which permits unrestricted use, distribution, and 
aimed to evaluate the clinical recurrences according to expression of endogenous IAP in colonic mucosa from patients with CD.

\section{Methods}

\section{Subjects and specimen collections}

We obtained biopsy tissue samples from the colonic mucosa during colonoscopy following informed consent (approved by the Ethics Committee of the Chonnam National University Hospital) in the 32 patients with CD. Biopsies were taken from grossly inflamed mucosa (edge of ulcer or aphthoid lesions if present), and from grossly non-inflamed mucosa in the same segment of colon. Colonic biopsy specimens were immediately snap-frozen in liquid nitrogen for protein analysis. Crohn's disease activity index (CDAI) and SES-CD were evaluated around the time of colonoscopy. We monitored the clinical courses of patients during follow-up after acquisition of biopsy specimens.

\section{Evaluation of IAP expression}

We performed quantification of IAP from each specimen using ELISA. We got colonic mucosal tissues using biopsy forceps and then homogenized those using the bead beater (QIAGEN Hilden, Germany) after protein extraction using the RIPA lysis buffer containing phosphatase inhibitor and protease inhibitor (sigma R0278, Sigma-Aldrich Co. LLC, USA). Protein concentrations of tissue lysates were quantitated by $\mathrm{BCA}^{\mathrm{Tm}}$ protein assay kit (ThermoFisher scientific, USA) with a standard (bovine serum albumin). Levels of IAP were measured by ELISA using a human IAP ELISA kit (Cloud-Clone Corp., Uscn Life Science Inc., Houston, USA) in which microtiter plate has been precoated with specific antibody to IAP. Absorbance was measured at $450 \mathrm{~nm}$ using a VersaMax ${ }^{\text {тM }}$ ELISA reader (MOLECULAR DEVICES, Silicon Valley, USA). We performed all reactions in duplicates and calculated the average value of duplicate results. The minimum detectable dose of IAP was typically less than 0.63 $\mathrm{ng} / \mathrm{mL}$.

\section{RNA isolation and real-time polymerase chain reaction}

To compare the degree of inflammation between the inflamed lesion and the noninflamed lesion, we determined the mRNA levels of pro-inflammatory genes such as IL-6, TNF- $\alpha$ and TLR-4 by real-time polymerase chain reaction (PCR) Taqman probe assay. Total RNA was isolated using $1 \mathrm{~mL}$ Trizol reagent (Takara Bio Inc. Japan) according to RNA extraction manufacturer's protocol. We subsequently converted total RNA to cDNA using the Reverse Transcription System (Promega, Wisconsin, USA). And then we analyzed mRNA of inflammatory factors by the real time PCR. We used Thermo Scientific ${ }^{\mathrm{TM}}$ commodified Taqman probe and primer set for inflammatory cytokine mRNA level detection by real time PCR. Catalogue number of Cytokines probe and primer set were HS02758991-g1:GAPDH, HS00174131-m1:IL-6, HS01113624-g1:TNF, HS001529 39-m1:TLR4(Thermo Fisher Scientific inc.). We detected the products using the ABI PRISM 7900HT Detection system (ThermoFisher scientific, USA). We calculated quantitative change of relative gene expression with $2^{-\triangle \Delta C T}$ method. The house keeping gene is glyceraldehyde phosphate dehydrogenase (GAPDH).

\section{Outcomes}

The primary outcome was clinical recurrence that was defined as change of prescription, bowel resection, fistulotomy, stricture plasty, stoma formation, CD-related hospitalization or flare during follow-up period. CD-related hospitalization was defined as cases resulting from complications including the following: CD-related surgery, hospitalization for nonsurgical CD-related events, such as CD-related flares; hospitalization related to complications/extraintestinal manifestation of $\mathrm{CD}$ and disease flare.

\section{Statistical analysis}

We used Wilcoxon signed rank test (one sample nonparametric test) to compare the expression of inflammatory markers and the IAP between inflamed mucosa and noninflamed mucosa in patients with CD. Mann-Whitney U test was used to compare the level of endogenous IAP between patients with clinical recurrence and patients without clinical recurrence during follow-up. The Statistical Package for the Social Sciences (SPSS)/PC 20.0 (Chicago, IL, USA) was used to perform the calculations. Differences were considered significant at $p<0.05$.

\section{Results}

\section{Demographics and baseline clinical characteristics}

A total of 32 consecutive patients (22 males) with CD were in clinical remission when they were included in the study. Median age (range) of patients was 22.5 years (15 years-49 years). Median CDAI (range) was 93.7 (22.8 154.9). All patients had undergone colonoscopy for surveillance of CD. There were colonic involvements in all patients and perianal involvement in $43.8 \%$ patients (Table 1).

\section{Expression of pro-inflammatory genes between the non-} inflamed mucosa and inflamed mucosa in patients with Crohn's disease

To verify the degree of inflammation between the inflamed and non-inflamed mucosa, we evaluated the mRNA levels of inflammatory genes. The mRNA levels of IL-6 ( $p=0.005)$ and TLR-4 $(p=0.013)$ in inflamed mucosa were significantly higher than those in non-inflamed 
Table 1 Demographics of 32 patients with Crohn's disease

\begin{tabular}{|c|c|c|c|c|}
\hline & $N=32$ & $\begin{array}{l}\text { Patients without clinical } \\
\text { recurrences }(n=13)\end{array}$ & $\begin{array}{l}\text { Patients with clinical } \\
\text { recurrences }(n=19)\end{array}$ & $p$-value \\
\hline Male, $n(\%)$ & $22(68.8)$ & $10(76.9)$ & $12(63.2)$ & 0.467 \\
\hline Age at inclusion, median (range), yrs. & $22.5(15-49)$ & $23.0(15 \sim 49)$ & $22.0(15 \sim 38)$ & 0.260 \\
\hline \multicolumn{5}{|l|}{ Clinical symptoms, $n(\%)$} \\
\hline diarrhea & $18(56.3)$ & $9(69.2)$ & $9(47.4)$ & 0.289 \\
\hline Abdominal pain & $13(40.6)$ & $5(38.5)$ & $8(42.1)$ & 0.837 \\
\hline Bloody stool & $8(25.0)$ & $2(15.4)$ & $6(31.6)$ & 0.299 \\
\hline Mucoid stool & $2(6.3)$ & $0(0.0)$ & $2(10.5)$ & 0.502 \\
\hline fever & $1(3.1)$ & $0(0.0)$ & $1(5.3)$ & 0.594 \\
\hline Weight loss & $3(9.4)$ & $1(7.7)$ & $2(10.5)$ & 0.999 \\
\hline \multicolumn{5}{|c|}{ Disease, phenotype, $n(\%)$, Montreal classification } \\
\hline L1 (isolated ileal type) & $0(0)$ & $0(0)$ & $0(0)$ & 0.473 \\
\hline L2 (isolated colonic type) & $12(37.5)$ & $6(46.2)$ & $6(31.6)$ & \\
\hline L3 (ileocolic type) & $20(62.5)$ & $7(53.8)$ & $13(68.4)$ & \\
\hline B1 (nonstricturing, nonpenetrating) & $4(12.5)$ & $1(7.7)$ & $3(15.8)$ & 0.528 \\
\hline B2 (strcituring) & $17(53.1)$ & $7(53.8)$ & $10(52.6)$ & \\
\hline B3 (penetrating) & $11(34.4)$ & $5(38.5)$ & $6(31.6)$ & \\
\hline P (concomitant perianal disease) & $14(43.8)$ & $4(30.8)$ & $10(52.6)$ & 0.221 \\
\hline \multicolumn{5}{|l|}{ Laboratory findings, median value (range) } \\
\hline WBC, median value (range) & $7100(2700-13,400)$ & $7100(5000-12,600)$ & $6900(2700-13,400)$ & 0.929 \\
\hline $\mathrm{Hb}$ & $12.9(9.0-16.0)$ & $13.6(10.3-16.0)$ & $12.9(9.0-16.0)$ & 0.421 \\
\hline Serum albumin & $4.1(3.0-5.0)$ & $4.1(3.4-5.0)$ & $4.1(3.0-5.0)$ & 0.453 \\
\hline C-reactive protein & $1.74(0.68-3.71)$ & $2.13(0.05-7.09)$ & $1.73(0.03-7.93)$ & 0.764 \\
\hline \multicolumn{5}{|l|}{ Medication, $n(\%)$} \\
\hline 5-Aminosalicylic acid & $28(87.5)$ & $11(84.6)$ & $17(89.5)$ & 0.542 \\
\hline Corticosteroid & $0(0.0)$ & $0(0.0)$ & $0(0.0)$ & 0.999 \\
\hline Azathioprine & $12(37.5)$ & $4(30.8)$ & $8(42.1)$ & 0.393 \\
\hline TNF- $a$ antagonists & $2(6.3)$ & $0(0.0)$ & $2(10.5)$ & 0.345 \\
\hline CDAl, median (range) & $93.7(22.8 \sim 154.9)$ & $84.9(22.8 \sim 154.9)$ & $95.9(46.1 \sim 147.1)$ & 0.387 \\
\hline SES-CD, median (range) & $14.0(4 \sim 37.0)$ & $13.0(4.0 \sim 31.0)$ & $14.0(4.0 \sim 37.0)$ & 0.486 \\
\hline
\end{tabular}

CDAI Crohn's Disease Activity Index, SES-CD Simplified endoscopic score of Crohn's disease, TNF- $a$ tumor necrosis factor alpha

mucosa. However, there was no difference of expression of TNF- $\alpha$ mRNA ( $p=0.345$, Fig. 1$)$.

\section{Clinical course in patients with Crohn's disease}

During a 14-month follow-up (range, 9 months-54 months), there were 19 patients with clinical recurrences. There were 9 patients who need a change of prescription, 3 patients who had undergone bowel resection, 6 patients who had undergone fistulotomy, and 5 patients who were hospitalized for complications/extraintestinal manifestation of $\mathrm{CD}$ and disease flare.

Table 1 showed the differences of prognostics between patients with clinical recurrences and patients without clinical recurrences. There were no significant differences in clinical symptoms, disease phenotype, baseline laboratory findings, CDAI and SES-CD between 2 groups.

Expression of endogenous IAP protein between inflamed mucosa and non-inflamed mucosa in patients with Crohn's disease

Mean (SD) concentrations of IAP in non-inflamed mucosa and inflamed mucosa were $26.3(16.8) \mathrm{ng} / \mathrm{mL}$ and $43.0(38.6) \mathrm{ng} / \mathrm{mL}$, respectively. There were no significant differences in concentration of IAP of non-inflamed mucosa and inflamed mucosa between patients with clinical recurrences and patients without clinical recurrences (both $p>0.05$ ). However, in patients without clinical recurrence, expression of IAP from inflamed mucosa was higher than that from non-inflamed mucosa $(p=0.019)$. 


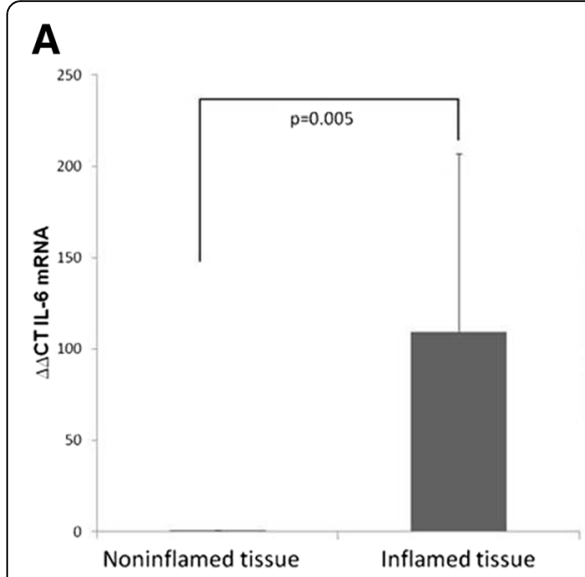

B

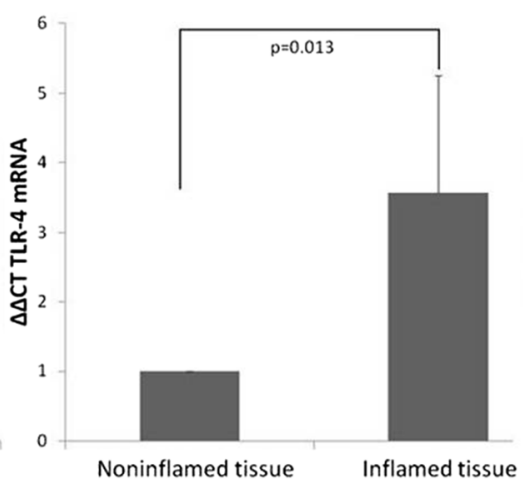

C

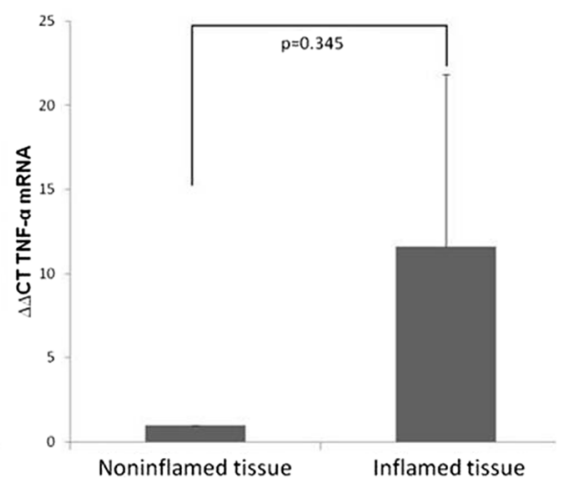

Fig. 1 Expression of pro-inflammatory genes between the inflamed mucosa and non-inflamed mucosa in patients with Crohn's diseases. Expression of mRNA levels of IL-6 (a) and TLR-4 (b) in inflamed mucosal were higher than those in non-inflamed mucosa. There was no significant difference in TNF-a (c)

In patients with clinical recurrence, there was no significant difference in IAP between noninflamed mucosa and inflamed mucosa (Fig. 2). There was no correlation between concentrations of IAP in non-inflamed mucosa and SES-CD $(r h o=-0.08, p=0.672)$. There was no correlation between concentrations of IAP in inflamed mucosa and SES-CD $($ rho $=-0.11, p=0.540)$.

The median ratio of IAP concentration of inflamed mucosa to non-inflamed mucosa in patients was 1.2 (range, $0.4-13.9$ ). There were 10 patients with IAP ratio $\leq 1.0$ and 22 patients with IAP ratio $>1.0$. There was a significant difference in clinical recurrences based on IAP ratio $(\leq 1.0)$. There were 9 patients $(9 / 19,47.4 \%)$ with IAP ratio $\leq 1.0$ in patients with clinical recurrence while there was one patient $(1 / 13,7.7 \%)$ with IAP ratio $\leq 1.0$ in patients without clinical recurrence $(p=0.024$, Fig. 2$)$.

\section{Discussion}

In this study, we showed that $31.3 \%$ patients had relatively lower IAP expression in inflamed colonic mucosa compared to noninflamed colonic mucosa. Among those, 90\% experienced clinical recurrences during follow-up.

There were inconsistent results for IAP activity in inflammatory bowel condition. IAP activity in inflamed tissue was marked upregulated in multiple models of colitis [7]. However, decreased IAP expression has been known to be involved in many chronic inflammatory diseases such as IBD, celiac disease, antibiotic associated diarrhea and necrotizing enterocolitis [8]. Furthermore, recent studies suggested a therapeutic role of human recombinant IAP in the management of chronic inflammatory bowel diseases [4, 9]. Therefore, we hypothesized that relative expression of IAP in inflamed mucosa compared to noninflamed mucosa may be associated with prognosis in patients with $C D$. In our study, 22 patients had higher expression of endogenous IAP in inflamed colonic mucosa compared to noninflamed colonic mucosa (ratio of endogenous IAP > 1.0). On the contrary, 10 patients had lower expression of endogenous IAP in inflamed colonic mucosa compared to noninflamed colonic mucosa (ratio of endogenous IAP $\leq 1.0$ ), which was similar with previous other studies $[4,10]$. We demonstrated that aggravation of underlying disease activity, which needed to change managements, had more frequently occurred in patients with low ratio $(\leq 1.0)$ of endogenous IAP expression than in patients with high ratio $(>1.0)$ of endogenous IAP. These finding suggest the role of endogenous IAP expression as prognostic factors for patients with $\mathrm{CD}$.

Clinical assessment is a critical for patients care, though several biomarkers and radiologic and endoscopic scoring system have a role to assess disease activity, determine prognosis, and predict response to therapeutic strategies. There are available use biomarkers including fecal calprotectin, C-reactive protein, erythrocyte sedimentation rate, peripheral antinuclear cytoplasmic antibody (p-ANCA), anti-Saccharomyces antibody (ASCA), and serum albumin in real clinical practice. In our study, we showed that there were no significant differences in laboratory findings, endoscopic scoring system, and CDAI between patients with clinical recurrence and patients without clinical recurrence [11]. Furthermore, there was no correlation between concentrations of IAP and clinical recurrence, which may limit the potential utility of absolute concentration of IAP as a prognostic marker. However, a low ratio of endogenous IAP $(<1.0)$ was correlated with poor prognosis in our study. Therefore, relative expression of IAP rather than absolute value of IAP may be more useful for prediction of prognosis.

There were several reports showing the effectiveness of exogenous IAP in management of IBD [6]. Several studies reported therapeutic effects of exogenous oral IAP in experimental colitis model [2, 12-14]. In colitic models, 

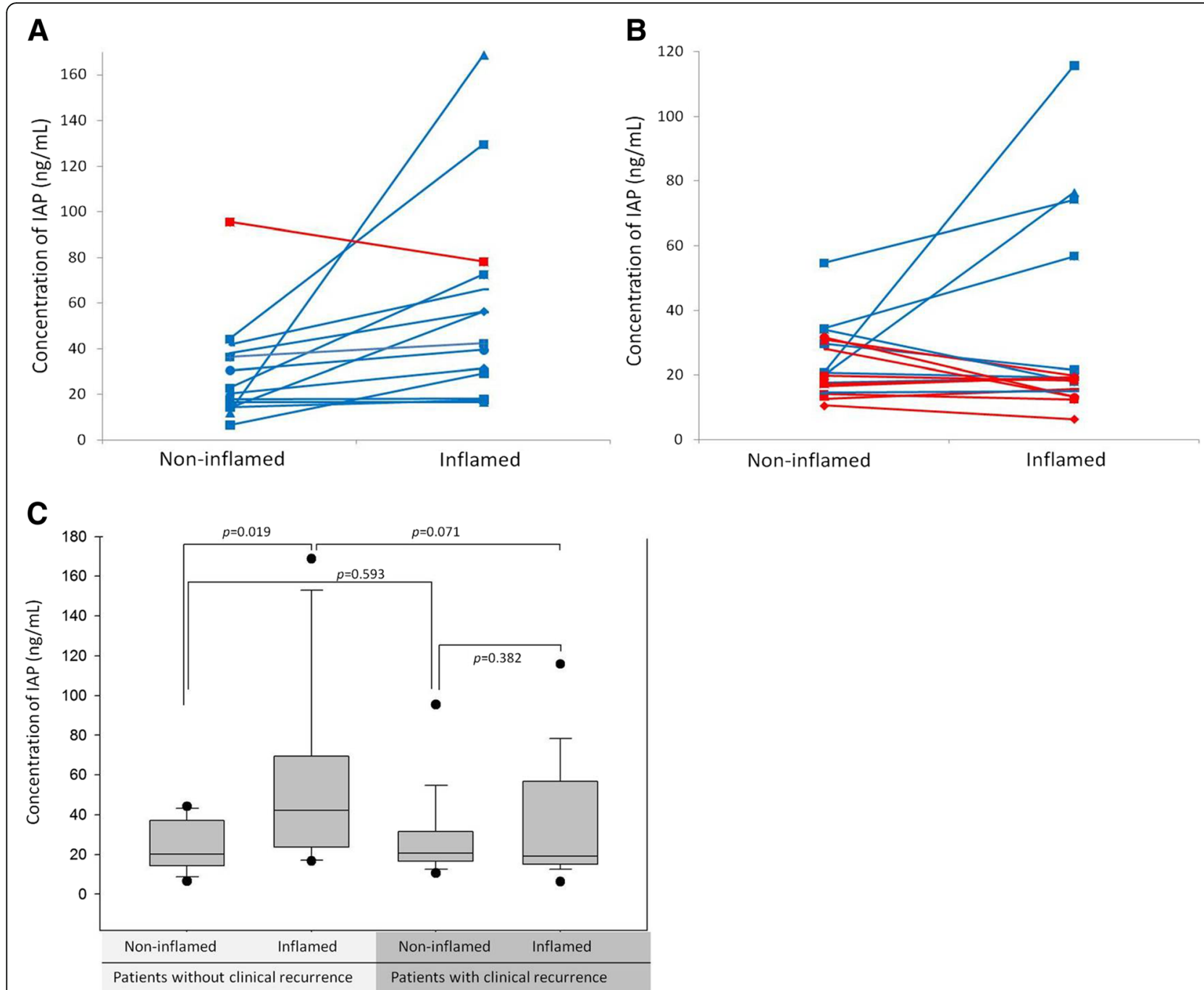

Fig. 2 Concentration of IAP in colonic mucosa from patients without clinical recurrence and with clinical recurrence. a Only one patient without clinical recurrence had lower concentration of IAP from inflamed mucosa compared to non-inflamed mucosa. b Among 19 patients with clinical recurrence, $47.4 \%$ patients had lower concentration of IAP from inflamed mucosa compared to inflamed mucosa. c In patients without clinical recurrence, expression of IAP from inflamed mucosa was higher than that from non-inflamed mucosa $(p=0.019)$. However, in patients with clinical recurrence, there was no significant difference in IAP between noninflamed mucosa and inflamed mucosa

exogenous administration of IAP improved the macroscopically and microscopically finding of colonic inflammation through inhibition of LPS-induced production of TNF- $\alpha$ and IL-6 and TLR-4 dependent pathway $[4,6,15,16]$. Administration of calf IAP by oral route prevented weight loss in IAP-knockout mice and dextran disulfate sodium induced colitis model. In patients with moderate to severe ulcerative colitis, enteral administration of IAP improved short-term clinical course [4]. Therefore, endogenous IAP may affect mucosal protection in patients with $\mathrm{CD}$. In our study, incidence of clinical recurrence was higher in patients with lower expression of endogenous IAP compared to patients with higher expression of endogenous IAP.

Limitations of our study were as follows; (1) most patients in our study had moderate disease activity during enrollment period. That is one of plausible reasons why the markers including concentration of IAP didn't predict the outcomes. Therefore, it will be difficult to impact current care practices with current results. (2) we couldn't get the information of IAP expression during clinical recurrence as ethical problem.

\section{Conclusions}

Relatively lower expression of IAP in inflamed mucosa compared to noninflamed mucosa may be associated with poor prognosis. In the future, it is necessary to perform study with larger sample size and variable disease activities to evaluate the role of IAP as a prognostic factor. And, the effectiveness of tailored treatment of IAP based on the expression of endogenous IAP need to be studied. 


\section{Acknowledgements}

No

\section{Funding}

This study was supported by the Chonnam National University Hospital Research Institute of Clinical Medicine (CRI 14011-1). This funding source had no role in the design of this study and collection, analysis and interpretation of data and in writing the manuscript.

\section{Availability of data and materials}

The datasets generated during and/or analyzed during the current study are available.

\section{Authors' contributions}

All authors have read and approved this manuscript. SYP and JYK contributed equally to this work. SYP review of clinical data, test results, interpretation of the results and drafting the manuscript. JYK acquisition and analysis of laboratory data. HSK Staff supervisor, review of patient data, test results and authorship. SML acquisition and analysis of laboratory data. JOC, JHS, SMK, DHK, CHP, JKJ, YEJ, JHL, SKC, JSR recruiting patients, review of clinical data and test results.

\section{Ethics approval and consent to participate}

Ethics Committee of the Chonnam National University Hospital approved this current study and we obtained informed written consent from the 32 patients with CD.

\section{Consent for publication}

Not Applicable.

\section{Competing interests}

The authors declare that they have no competing interests.

\section{Publisher's Note}

Springer Nature remains neutral with regard to jurisdictional claims in published maps and institutional affiliations.

\section{Author details}

${ }^{1}$ Division of Gastroenterology and Hepatology, Chonnam National University Medical School, Gwangju, South Korea. ${ }^{2}$ Division of Endocrinology and Metabolism, Chonnam National University Medical School, Gwangju, South Korea. ${ }^{3}$ Department of Surgery, Chonnam National University Medical School, Gwangju, South Korea. ${ }^{4}$ Department of Pathology, Chonnam National University Medical School, Gwangju, South Korea. ${ }^{5}$ Department of Internal Medicine, Chonnam National University Medical School, 42, Jaebongro, Dong-ku, Gwangju 501-757, Korea.

Received: 20 August 2018 Accepted: 8 November 2018 Published online: 17 December 2018

\section{References}

1. Alpers DH, Zhang Y, Ahnen DJ. Synthesis and parallel secretion of rat intestinal alkaline phosphatase and a surfactant-like particle protein. Am J Phys. 1995;268(6 Pt 1):E1205-14.

2. Lalles JP. Intestinal alkaline phosphatase: novel functions and protective effects. Nutr Rev. 2014;72(2):82-94.

3. Bilski J, Mazur-Bialy A, Wojcik D, Zahradnik-Bilska J, Brzozowski B, Magierowski M, Mach T, Magierowska K, Brzozowski T. The role of intestinal alkaline phosphatase in inflammatory disorders of gastrointestinal tract. Mediat Inflamm. 2017;2017:9074601.

4. Tuin A, Poelstra K, de Jager-Krikken A, Bok L, Raaben W, Velders MP, Dijkstra G. Role of alkaline phosphatase in colitis in man and rats. Gut. 2009;58(3): 379-87.

5. Molnar K, Vannay A, Szebeni B, Banki NF, Sziksz E, Cseh A, Gyorffy H, Lakatos PL, Papp M, Arato A, et al. Intestinal alkaline phosphatase in the colonic mucosa of children with inflammatory bowel disease. World J Gastroenterol. 2012;18(25):3254-9.

6. Ramasamy S, Nguyen DD, Eston MA, Alam SN, Moss AK, Ebrahimi F, Biswas B, Mostafa G, Chen KT, Kaliannan K, et al. Intestinal alkaline phosphatase has beneficial effects in mouse models of chronic colitis. Inflamm Bowel Dis. 2011;17(2):532-42
7. Sanchez de Medina F, Martinez-Augustin O, Gonzalez R, Ballester I, Nieto A, Galvez J, Zarzuelo A. Induction of alkaline phosphatase in the inflamed intestine: a novel pharmacological target for inflammatory bowel disease. Biochem Pharmacol. 2004;68(12):2317-26.

8. Fawley J, Gourlay DM. Intestinal alkaline phosphatase: a summary of its role in clinical disease. J Surg Res. 2016;202(1):225-34.

9. Lukas $M$, Drastich $P$, Konecny $M$, Gionchetti $P$, Urban O, Cantoni F, Bortlik M, Duricova D, Bulitta M. Exogenous alkaline phosphatase for the treatment of patients with moderate to severe ulcerative colitis. Inflamm Bowel Dis. 2010; 16(7):1180-6.

10. Molnar K, Vannay A, Sziksz E, Banki NF, Gyorffy H, Arato A, Dezsofi A, Veres $G$. Decreased mucosal expression of intestinal alkaline phosphatase in children with coeliac disease. Virchows Arch. 2012;460(2):157-61.

11. Lichtenstein GR, McGovern DPB. Using markers in IBD to predict disease and treatment outcomes: rationale and a review of current status. Am J Gastroenterol Suppl. 2016;3:17

12. Hamarneh SR, Mohamed MM, Economopoulos KP, Morrison SA, Phupitakphol T, Tantillo TJ, Gul SS, Gharedaghi MH, Tao Q, Kaliannan K, et al. A novel approach to maintain gut mucosal integrity using an oral enzyme supplement. Ann Surg. 2014;260(4):706-14.

13. Keswani SG, King A. Intestinal alkaline phosphatase prevents the systemic inflammatory response associated with necrotizing enterocolitis. J Surg Res. 2013;185(1):e9-10.

14. Chen KT, Malo MS, Beasley-Topliffe LK, Poelstra K, Millan JL, Mostafa G, Alam SN, Ramasamy S, Warren HS, Hohmann EL, et al. A role for intestinal alkaline phosphatase in the maintenance of local gut immunity. Dig Dis Sci. 2011 . 56(4):1020-7.

15. Hwang SW, Kim JH, Lee C, Im JP, Kim JS. Intestinal alkaline phosphatase ameliorates experimental colitis via toll-like receptor 4-dependent pathway. Eur J Pharmacol. 2018;820:156-66.

16. Bol-Schoenmakers M, Fiechter D, Raaben W, Hassing I, Bleumink R, Kruijswijk D, Maijoor K, Tersteeg-Zijderveld M, Brands R, Pieters R. Intestinal alkaline phosphatase contributes to the reduction of severe intestinal epithelial damage. Eur J Pharmacol. 2010;633(1-3):71-7.
Ready to submit your research? Choose BMC and benefit from:

- fast, convenient online submission

- thorough peer review by experienced researchers in your field

- rapid publication on acceptance

- support for research data, including large and complex data types

- gold Open Access which fosters wider collaboration and increased citations

- maximum visibility for your research: over $100 \mathrm{M}$ website views per year

At BMC, research is always in progress.

Learn more biomedcentral.com/submission 